\title{
PERSPECTIVA HISTORICA \\ DE LOS MODELOS ARIMA Y SU UTILIDAD \\ EN EL ANALISIS ECONOMICO
}

ANTONI ESPASA

Universidad Carlos III

Con el trabajo de J. Fourier en 1807, en el que se demuestra que una serie temporal se puede aproximar tanto como se quiera mediante la suma de términos de senos y cosenos, podemos decir que nace el Análisis de Series Temporales (AST). No obstante, esta cxpansión de Fourier es solamente válida para series determinísticas $y$, durante todo el siglo XIX y primeros años del $\mathrm{Xx}$, en el AST el enfoque determinístico fuc el único que prevaleció. En tal contexto, la discrepancia entre los valores de los modelos y los datos reales se atribuía a un elemento residual estocástico.

Siguiendo a Yaglom (1962), p. 4, podemos decir que los primeros intentos de introducir un enfoque estocástico en el AST se encuentran en Bachelier (1912), pero fue en los años treinta, con los trabajos de Slutski (véase Slutski, 1960), Kolmogorov (véase Kolmogorov, 1931 y 1950) y Kinchin (1934), cuando empieza a construirse una teoría general sobre procesos estocásticos. Por otro lado, en Yule (1926 y 1927) se señala que el análisis de Fourier no es adecuado para series reales, pues en ellas las amplitudes y períodos de los componentes sinuosidales son estocásticos, y que tal faceta se puede recoger expresando una serie en función de sus valores pasados. En concreto, Yule (1927) introduce y desarrolla los procesos autorregresivos de segundo orden como esquemas teóricos capaces de generar series con oscilaciones cíclicas estocásticas. La generalización del análisis de Fourier para funciones estocásticas no se obtuvo hasta los años cuarenta con dos trabajos independientes de Kolmogorov (1940) y Crámer (1942), que constituyen el soporte teórico del moderno análisis espectral y establecen la correspondencia entre el enfoque de las covarianzas (o del dominio del tiempo) y el enfoque espectral (o del dominio de las frecuencias) en el estudio de los procesos estocásticos estacionarios. Previamente, el proceso de Yule fue generalizado por Walker (1931) introduciendo un esquema general de procesos autorregresivos, y Slutski (1937) establecía que series temporales con oscilaciones cíclicas podían también venir generadas por otro tipo de procesos e introdujo los procesos de medias móviles. La integración de los procesos de Yule y Slutski se realizó con el trabajo de 
Wold (1938), donde se establece una formulación general para cualquier tipo de proceso estocástico estacionario y se introducen los procesos mixtos, autorregresivos y de medias móviles (ARMA).

Este desarrollo teórico sobre procesos estocásticos se ciñó a procesos estacionarios; sin embargo, desde finales del siglo XIX, véanse Poynting (1844) y Hooker $(1901)^{1}$, se tcnía asimilado en el análisis empírico que las series reales podían contener, además de oscilaciones cíclicas, un componente tendencial. De hecho, esta descomposición de una serie temporal en tendencia, componentes cíclicos y elemento residual se fue popularizando cn las aplicaciones económicas hasta culminar en el trabajo de Shiskin et al. (1967), en que se presenta un procedimiento de extracción de señales $(\mathrm{X}-11)$, por el que una serie se descompone en tendencia, componente estacional y elemento irregular. En estos procedimientos de descomposición de series temporales, los componentes de las mismas fueron, desde los años veinte, considerados como estocásticos y su estimación se basaba en aplicar medias móviles a los datos originales. De esta forma empiricista se suplía la falta de una teoría sobre procesos estocásticos evolutivos.

En el campo de la predicción también se desarrollaron procedimientos empiricistas para abordar con cierto éxito la predicción de series temporales con tendencia. Así surgieron los denominados procedimientos de alisado; véanse Holt (1957), Brown (1959), Holt et al. (1960), Winter (1960), Harrison (1965), etc. ${ }^{2}$.

En resumen, podeinos decir que al final de los sesenta el análisis aplicado de Series Temporales asumía plenamente que, en general, las series reales cran no estacionarias y para predecir y extraer señales en una serie concrcta se utilizaban procedimientos que tenían en cuenta tal faceta de las series y, además, se operaba de forma que tanto el componente estacionario como el evolutivo eran estocásticos. Junto con esto, el análisis teórico sólo había desarrollado un esquema estocástico general para el componente estacionario de las series temporales y, así, en aplicaciones más académicas era frecuente la práctica de eliminar previamente (véase Nelson y Ploser, 1982) tendencias determinísticas.

El puente entre los desarrollos teóricos y los procedimientos aplicados se ha tendido con la introducción por Box y Jenkins (1970) de los modelos ARIMA. Estos modelos generalizan los modelos ARMA permitiendo que no sea la serie original, sino una versión de la misma debidamente diferenciada, quien venga determinada por un modelo estacionario ARMA. Es decir, Box y Jenkins introducen un contexto teórico en el que las series

1 Referencias tomadas de Makridakis (1976); para un mayor detalle bibliográfico, véase la sección III.4 de dicho artículo

2 Para un mayor detaile bibliográfico, véasc Makridakis (1976), sección III.3. 
pueden ser evolutivas, pues no son sus niveles, sino sus incrementos o los incrementos de éstos, quienes tienen un comportamiento estacionario. En términos algebraicos, esto supone permitir raíces unitarias en la parte autorregresiva del modelo ARMA. Claramente, la evolutividad que consideran Box y Jenkins no es una evolutividad general, sino una evolutividad específica, que se puede denominar homogénea, y que sirve para explicar el comportamiento de series temporales que, pudiendo ser no estacionarias, su velocidad de avance o su aceleración tienen un comportamiento estacionario. Esta estacionariedad en la primera o segunda derivadas es la que confiere un carácter relativamente homogéneo a las series generadas por modelos ARIMA. Enfocando el problema a partir de la transformación (diferenciación) de la seric real que es estacionaria, tenemos que mediante la aplicación, una o dos veces, de un procedimiento de suma, integración (que es la transformación inversa a la diferenciación), de la serie estacionaria se llega a la serie real; de ahí el nombre de modelos Auto Regresivos Integrados y medias móviles (Moving Averages).

Como hemos señalado, los modelos ARIMA sólo son aptos para explicar una evolutividad de tipo homogéneo, pero Box y Jenkins intuyeron que ésta era una forma útil de aproximar la realidad evolutiva que observamos. De hecho, hoy en día sabemos que, si nos ceñimos a esquemas lineales, los procedimientos de alisado utilizados para predecir (véase Granger y Newbold, 1977) son óptimos cuando se aplican a series que siguen un tipo concreto de modelo ARIMA. Asimismo, sabemos (véase Cleveland y Tiao, 1976) que el procedimiento de $\mathrm{X}-11$ es óptimo cuando se aplica a series generadas por un determinado modelo ARIMA. Es decir, los modelos ARIMA suponen una generalización de los esquemas teóricos implícitos en los procedimientos empíricos que, previamente al libro de Box y Jenkins, se habían mostrado útiles y relativamente satisfactorios. Esta generalización a nivel univariante lograda por los modelos ARIMA ha determinado que en la investigación sobre extracción de señales, que mayoritariamente se enfoca como un problema univariante, se hayan desarrollado con éxito procedimientos de descomposición de Series Temporales que se basan en modelos ARIMA: a) el modelo ARIMA de la serie observada -procedimientos de forma reducida-: Burman (1980), Hillmer y Tiao (1982), Maravall (1987)...; b) los modelos ARIMA postulados para los componentes - procedimientos estructurales-: Engle (1978), Harvey y Todd (1983), Fernández (1988)...

La contribución de Box-Jenkins no fue meramente teórica, sino enormemente práctica, pues, junto con el esquema teórico de modelo estocástico, propusieron una metodología para que sean los datos quienes, en cada caso, determinen el modelo ARIMA más adecuado para explicar su generación. Esto supone un progreso importante en el trabajo aplicado de predic- 
ción y extracción de señales, ya que no se utiliza un procedimiento de caja negra, más o menos basado en la experiencia analista, sino un procedimiento validado por los datos y que corresponde a un modelo del tipo ARIMA, que es un esqucma que permite una explicación general de la evolutividad humogénea.

Hay que señalar que la derivación de un esquema general para explicar un proceso evolutivo cualquiera será necesariamente una labor poco operativa en un mundo real en el que no se pueden replicar series temporales de un mismo proceso y, en consecuencia, los grados de libertad para estimar el comportamiento del proceso son limitados. Por ello, nos vemos obligados a trabajar con esquemas que meramente aproximen los verdaderos modelos teóricos bajo hipótesis específicas.

Así, si el mundo real cumpliese las condiciones de lineariedad y cvolutividad homogénea, los modelos ARIMA constituirían un esquema general para explicar el comportamiento de una serie en función de su pasado. Box y Tiao (1975) han ampliado los modelos ARIMA con la introducción de variables artificiales, generalmente binarias, dando lugar a la clase de mode. los denominados ARIMA con ANALISIS DE INTERVENCION, que son aptos para explicar series temporales que contengan, además de su evolución estocástica, determinados movimientos bruscos o atípicos.

La generalización que supone un modelo ARIMA tiene dos puntos débiles, ya que no es válida para un universo no lineal y sólo contempla una evolutividad de tipo homogéneo. Respecto la no linealidad hay que señalar que un esquema general sobre la misma, véase Weiner (1958), ha de ser necesariamente no operativo $y$, por tanto, una actitud más realista es pretender determinar contextos no lineales específicos, pero suficientemente amplios, para que sean capaces de aproximar la generación de cualquier seric real de forma más adecuada que los modelos lineales. Los umodelos dependientes del estado", introducidos por Priestley (1981), son modelos no lineales que van en la dirección mencionada y que contienen como casos particulares a los modelos bilincales, autorregresivos por zonas (Thresbold autoregresive) y autorregresivos exponenciales. No obstante, no existe todavía una metodología debidamente desarroilada para la aplicación de procedimientos no lincales; por ello, en el resto de este trabajo nos ceñiremos a modelos lineales.

La extensión de la evolutividad homogénea se ha logrado a nivel teórico, por ejemplo, con los modelos ARIMA de DIFERENCIACION FRACCIONAL [véase Granger y Joyeux (1980) y referencias previas allí mencionadas], pero las aplicaciones realizadas no han llevado, generalmente, a mejoras sustanciales sobre los modelos ARIMA, por lo que tampoco los consideraremos en el resto del trabajo. 
La caracterización de la evolutividad a través de las raíces unitarias es también el procedimiento empleado en Econometría, con la teoría de la cointegración; véanse Granger (1981 y 1986), Engle y Granger (1987), Escribano (1990), ICE (1990), Dolado (1990), etc. De hecho, el empleo del mismo tratamiento en cuanto a la evolutividad de las series económicas y la generalización estacionaria lineal que tecogen los modelos ARIMA es lo que determina el resultado, comentado más adelante, de que los modelos ARIMA son formas finales de los modelos econométricos simultáneos lineales.

$\mathrm{Si}$, bajo las restricciones señaladas, hemos concluido que los modelos ARIMA con ANALISIS DE INTERVENCION constituyen una clase general para explicar series económicas en función exclusivamente de su propio pasado, conviene estudiar cuál es su relación con un modelo global que contemple la determinación multivariante de la serie en cuestión junto con todas aquellas con las que está relacionada.

A nivel econométrico, el modelo lineal global por excelencia es el modelo econométrico estructural (SEM, structural econometric model). desarrollado a principios de los años cincuenta en el seno de la Cowles Foundation (véase Koopmans y Hood, 1953) y dinamizado de forma paramétrica en Sargan (1959 y 1961) y Hendry (1976), y de forma semiparamétrica en el componente residual en Hannan y Terrell (1973), y globalmente en Espasa y Sargan (1977) y Espasa (1977). La relación de los modelos ARIMA univariantes con el modelo SEM fue inicialmente tratada por Zellner y Palm (1974) y ha sido posteriormente desarrollada por Prothero y Wallis (1976), Wallis (1977), Zcllner (1979) y Wallis (1980). El resultado que se desprende de esos trabajos consiste en que si las variables exógenas del modelo vienen determinadas por un modelo ARIMA multivariante ${ }^{3}$, cada una de las variables endógenas del modelo SEM viene determinada individuaimente por un modelo ARIMA.

Este resultado es de gran relevancia práctica. En efecto, dado que al modelo ARIMA univariante de una serie concreta se llega a partir del modelo global SEM, tenemos que en la medida que el modelo SEM refleja las características del mundo real, el modelo ARIMA de una seric individual incorpora, de forma ineficiente pero ciertamente de modo consistente, las características básicas de dicha scric.

En consecuencia, un modelo ARIMA no es una caja negta, ni un modelo ad hoc de mayor o menor utilidad en la predicción, sino que es mucho más: un modelo correcto para describir a nivel univariante el comporta-

\footnotetext{
3 Los modelos ARIMA multivariantes (véase Tiao y Box, 1991) son una genctalización lc los modelos multivariantes utilizados por Quenouille (1957).
} 
miento y características fundamentales de un fenómeno económico. Por tanto, un modelo ARIMA es una herramienta útil para hacer análisis económico, pues, si el modelo es correcto, sus resultados no pueden estar en contradicción con los que se deriven del modelo SEM. Obviamente, el análisis económico que se puede realizar a partir de un modelo ARIMA es enormemente limitado, pues ignora la relación de la variable en cuestión con-el resto de variables que configuran el universo económico y, en consecuencia, no se pueden realizar con él análisis de control, simulación, ni estructural, pero se puede realizar un análisis sobre las características básicas del fenómeno, que es plenamente válido.

Una aplicación del tipo de análisis económico que se puede realizar con un modelo ARIMA se enuncia en Espasa (1990) y se puede resumir como sigue. El modelo ARIMA contiene una descripción adecuada del comportamiento a largo plazo del fenómeno en cuestión y, a su vez, incorpora la estructura temporal con la que dicho fenómeno tiende a volver en cada momento a su sendero de crecimiento equilibrado (stady state), que es estocástico en el sentido de que viene determinado por las condiciones iniciales del sistema.

A nivel univariante, el interés de disponer de un instrumento que sirviera para interpretar y caracterizar la variable en cuestión ha sido durante mucho ticmpo ambiguo, o inexistente, debido fundamentalmente a que tanto los procedimientos de predicción basados en el alisamiento de series como los de descomposición en tendencia, componentes cíclicos y elemento residual no se apoyaban en una teoría univariante de aceptación general, ni estaban vinculados de forma directa con el modelo SEM, que es el esquema reconocido en la profesión como válido para representar el universo económico. Sin embargo, los modelos ARIMA incorporan una teoría univariante bastante general y se derivan de los modelos SEM, por lo que podemos concluir que sirven para caracterizar una serie económica. Un procedimiento de aplicación automática de los modelos ARIMA con Análisis de Intervención para caracterizar un conjunto amplio de series temporales se encuentra en Revilla et al. (1990). 


\section{BIBLIOGRAFIA}

Bachelrer, L. (1912): Calcul des Probabilités, Paris, Garthicr-Villars.

Box, G. E. P., y Jenkins, G. M. (1970): Time Series Analysis, Forecasting and Control. Holden Day.

BOX, G. E. P., y 'TıAO, G. C. (1975): "Intervention Analysis with applications to economic and environmental problems", $J$. of the American Statistical Association, vol. 70, núm. 349, PP. 70.79 .

BRown, R. G. (1959): Statistical Forecasting for Inventory Contml, Nucva York, McGraw-Hill.

Burman, J. P. (1980): "Seasonal Adjustment by Seasonal Extraction", J. of the Royal Stat. Soc. A., 143, PP. 321.337.

Cleveland, W. P., y Tiao, G. C. (1976): "Decomposition of Seasonal Time Series: A model of the Census X-II Program", J. of the American Statistical Association. vol. 71 , núm. 355 (septiembrc), pp. 581-587.

ĆÁmER, H. (1942): "On harmonic analysis of certain tunction spaces", Arkit. Mat. Astrom. Fysik, 288 (12), $1-7$.

DOLADO, J. J. (1990): "Cointegración: una panorámicin, Estadtstica Española, vol. 32, núm. 124 (mayo-agosto), pp. 327-365.

ENGle, R. F. (1978): "Estimating Structural Modcls of Scasonality", cn Seasonal Analyst. of Economit Time Series, ed. A. Zellnet, Washington D. C., U. S. Dept. of CommerceBureau of the Census, Pp. $281-297$.

ENGLE, R. F., y GRANGER, C. W. J. (1987): "Cointegration and Error Correction: Represcntation, Estimation and 'Testing", Econométrica, vol. 55, núm. 2 (marzo). Traducción al castellano publicada en ICE (1990)

Escribano, A. (1990): "Introducción al tema de cointegración y tendencias", Cuademos Económicos, núm. 4, pp. 7-42.

EspasA, A. (1977): The Spectral Maximun Likelibond Estimation of Eonometric Modeli with Stationary Errors, Göttingen, Vandenhocck and Ruprecht.

- (1990): "Metodología para el análisis de la coyuntura de un fenómeno económico", Banco de España, documento de trabajo 9003.

Espasa, A., y SARGaN, J. D. (1977): "The Spectral Estimation of Simultancous Equation Systems with Lagged Endogenous Variables", Intemat. Eco. Rez, vol. 18, num. 3.

FrrnánDez, F. J. (1988): "Modelos estructurales de Series Temporales: una aplicación al análisis y predicción de agregados monetarios y fiscales". Revista de Economia Pablia. 1 , PP. 44-65.

Granger, C. W. J. (1981): "Some Properties of Time Scries Data and theit use in Econo. metric Model Specificationn, J. of Econometrics, 16 (11), pp. 121-130.

- (1986): "Developments in the Study of Cointegrated Economic Variables", Oxfort Bull of Eco. and Stat., 48,3 .

Granger, C. W. J., y Newbold, P. (1977): Forecasting Economic Series. Nueva York, Academic Press.

GRANGER, C. W. J., y JOYEe;X, R. (1980): "An introduction to long-memory time scries models and fractional difterencing", f. of Time Senes Analysi, vol. 1, núm. 1. pp. 15-29.

HannaN, E. J., y Terrfi.l, R. D. (1973): "Multiple Equation Systems with Stationary Errors", Eionométria, \&1, PP. 299-320.

HARRISON, P. J. (1965): "Short-Term forecasting", Applied Statistich, vol 14, pp. 102-139.

HARveY, A. C., y ToDn, P. H. J (1983): "Forecasting Economic Time Scrics with Structural and Box-Jenkins Models: A Case Study", J. of Busmess and Ein. Stat. 1, 4. pp. 290306.

HENDrY, D. F, (1976): "The Structure of Simulaneous Equations Estimatiors", /. of Eomometrics, 4, PP. 51.85

Hillamer, S. C., y TIAO, G. C. (1982): "An ARIMA-Model Based Approach to Scasonal Adjustrient", f. of the American Stat. Ass. 77. Pp. 63-70. 
Horr, C. C. (1957): "Forecasting seasonal and trends by exponentially weighted moving averages», Pittsburgh (Pennsylvania), Carnegic Institute of Technology.

HoLT, C. C. at al. (1960): Planning production inventories and Work Force, Nucva York, Pren tice-Hall.

HoOkEK, R. H. (1901): "The suspension of the Berlin produce exchange and its effect upon corn prices", J. of the Royal Statistical Society, vol. 6.t, pp. 574 y ss.

ICE, INFORMACIÓN COMERCiAL ESPAÑola (1990): "Cointegración y raíces unitarias: un área de crecimicnto". Cuademos Económicos, número monográfico, núm. 4.

KhiNeniN, A. Y. (1934): "Korrelationstheorie der stationaren stochastischen Prozesse", Mathematical Annals, 109, 604.

Kolmogorov, A. N. (1931): "Uber dic Abalytischen Methoden in der Wahrscheinlichkeitsrcchnung», Mathematical Annals, 104, 415.

- (1940): "Kurven in Hibertschen Raum die gegenuber eine einparametrigen Gruppe von Bewegugen invariant sind", C. R. (Doklady) de l'Academie des Sciences de l'URSS. New Series, $26,6-9$.

-- (1941a): "Stationarty sequences in Hilbert Space", Bul. Moscou State Univ, 2, núm. 6.

-- (19416): "Interpolation and extrapolation of stationary random sequences", Izv". Akad. Nauk. SSSR, Ser. Mal. 5, 3.

- (1950): Foundation of the Theory of Probability, Nucva York, Chelsca Publishing Company.

KoOPMANS, T. C., y HOOD, W. M. (1953): "The estimation of simultaneous linear cconomic realtionships", capítulo VI del libro Studies in Econometric Methids, monografía núm. 1/4 de la Cowles Foundation, editado por W. M. Hood y T. C. Koopmans.

Maravai.., A. (1987): "Descomposición de Series Temporales, especificación, estimación e inferencian, Estadística Española, núm. 11.4, pp. 11-69.

Neison, C. R., y PIossek, C. I. (1982): "Trends and random walks in macrocconomic times scrics", J. of Montary Economics, 10, pp. 139-162.

MAKRIDAkis, S. (1976): "A Survey of Time Scries", Int. Stat. Rev, vol, 44, núm. 1, pp. 2970.

PorNinc, J. H. (1884): "A comparison of the fluctuations in the price of wheat and in the cotton and silk imports into Grat Britain", J. of the Royal Statistical Sociecy, vol. 47, pp. 345-364.

Prinstiey, M. B. (1981): Spectral Analysis and Time Series, Academic Press.

Prothero, D. L., y Wallis, K. F. (1976): "Modelling Macrocconomic Time Scries", J. of the Rojal Statistical Society. Serie A, 139, part 4, pp. 468-485.

Quenounte, M. H. (1957): The Analysis of Multiple Time Series, Londres, C. Griffin and Co.

Rivilla, P.; RfY, P., y ESPASA, A. (1990): "Characterization of Production in Different Branches of Spanish Industrial Acrivity, by meany Time Series Analysis", trabajo no publicado, de próxima aparición como documento de trabajo del Departamento de Economía de la Universidad Carlos III.

SARGAN, J. D. (1959): "The Estimation of Relationships with Autocorrclated Residuals by the Lisc of Instrumcotal Variables", J. of the Royal Statistical Soc., B, 21, pp. 91-105.

- (1961): "The Maximum Likelihood Estimation of Economic Rclationships with Autoregresive Residuals", Econométrica, vol. 29, núm. 3 (julio).

Shiskin, J; Young, A. H., y Muschave, J. C. (1967): "The X-11 variant of the Census Method II Seasonal Adjustment Program", Bureau of the Census Technical puper n. 15 (revisado), Washington D. C., V. S. Department of Commerce.

SLLSTKI, E. (1937): "The summation of random causes as the source of cyclic processes", Econemétrica, vol. 5, pp. $105-146$.

- (1960): Selected Works: Probability Theory. Matbematical Statistics, Moscú, Izv. Akad. Nauk. SSSR.

'TAAO, G. C., y BOX, G. E. P. (1981): "Modeling multiple time series with applications", $J$. of the American Statistical Association, vol. 76, num. 376 (diciembre), pp. 802 y ss. 
Walker, A. M. (1931): "On the periodicity in series of related terms", Procedings of the Royal Society of London. A, 131, pp. 518-532.

Wall.LS, K. F. (1977): "Multiple Time Series Analysis and the Final Form of Econometric Models", feconmétria, vol. 48, num. 1.

-.. (1980): "Econonetric Implications of the Rational Expectations Hyphotesis", Econometri(a. vol. 48 , núm. 1

WIENER, N. (1950): Extrapolation, Interpretation and Smothing of Stationary Time Series. Nuevat York, MIT Technology Press and John Wilcy and Sons, Inc.

- (1958): Non-linear problems in random theory, Cambridge (Mass.), MIT Press

WINTERS, P. R. (1960): "Forecasting Sales by exponentially weighted moving averages", Management Sicne, abril, pp. 324-342.

Wolls (1938): A study in the Analysig of Stationary Time Serits. Uppsala. Alinquist and Wiksells.

Yagiom, A. M. (1962): An Introduction to the Theory of Stationary Random Iituntions, Nueva York, Dover Publications, Inc.

YULE, G. U. (1926): "Why do we sometimes get nonsense-corrclations between time series? A study in sampling and the nature of time series". I. of the Royal Statistical Soctoty. 89, $1-64$

- (1927): "On the method of investigating periodicities in disturbed series, with special reference to Wolfer's sunspot numbers", Philosopbical Transattions, A., 226, pp. 267-298.

Zeidiner, A., y PALM, F. (1974): "Time Series Analysis and Simulaneous Equation Econometric Models", J. of Econometrics, vol. 2, núm. 1 (mayo), pp. 17-54.

Zfiner, A. (1979): "Statistical Analysis of Econometric Models", J. of the American Statistical Association, vol. 74, núm. 367 (septicmbre), pp. 628-65I. 